\title{
Visualizing Underwater Ocean Optics
}

\author{
Diego Gutierrez, Francisco J. Seron, Adolfo Munoz and Oscar Anson
}

University of Zaragoza, Spain

\begin{abstract}
Simulating the in-water ocean light field is a daunting task. Ocean waters are one of the richest participating media, where light interacts not only with water molecules, but with suspended particles and organic matter as well. The concentration of each constituent greatly affects these interactions, resulting in very different hues. Inelastic scattering events such as fluorescence or Raman scattering imply energy transfers that are usually neglected in the simulations. Our contributions in this paper are a bio-optical model of ocean waters suitable for computer graphics simulations, along with an improved method to obtain an accurate solution of the in-water light field based on radiative transfer theory. The method provides a link between the inherent optical properties that define the medium and its apparent optical properties, which describe how it looks. The bio-optical model of the ocean uses published data from oceanography studies. For inelastic scattering we compute all frequency changes at higher and lower energy values, based on the spectral quantum efficiency function of the medium. The results shown prove the usability of the system as a predictive rendering algorithm. Areas of application for this research span from underwater imagery to remote sensing; the resolution method is general enough to be usable in any type of participating medium simulation.
\end{abstract}

Categories and Subject Descriptors (according to ACM CCS): I.3.7 [Computer Graphics]: Three-Dimensional Graphics and Realism

\section{Introduction}

Ocean water is arguably the richest participating medium in terms of optical thickness and the number and type of interactions that occur in it. This paper deals with the physicallybased rendering of underwater scenes by simulating the inwater light field, based on a compact bio-optical model that takes into account the dissolved and particulate matter, optically influential constituents of the water. To ensure accuracy, we use published data obtained from a wide range of literature in the field of oceanography. Our model is not restricted to just the visible spectrum and can be adapted to any type of known ocean water in particular, or to any kind of participating medium in general.

Scattering in water is caused by interactions of light at molecular level and with particles [Mob94]. It can be classified in two broad categories: elastic or inelastic scattering, depending on whether the scattered photon maintains or changes its energy in the process. The inelastic scattering events can be further subclassified according to the nature of the energy transfer: Stokes scattering, when a molecule of the medium absorbs the photon and re-emits it with a lower en- ergy, and anti-Stokes scattering, when the re-emitted photon has a higher energy. Both cases are covered by our model. The process implies an energy transfer from wavelength $\lambda^{\prime}$ to $\lambda$, with $\lambda^{\prime}$ being the excitation wavelength and $\lambda$ the reemitted wavelength. The former case implies a shift towards longer wavelengths, whereas in the latter the scattered photon has a shorter wavelength. Major forms of elastic events in water include Einstein-Smoluchowski scattering (see Section 3.2), whereas for inelastic events, Raman scattering and fluorescence are the two most prominent (see Section 3.3).

The presence and concentrations of the constituents in the water determine its optical properties. These optical properties are divided in two classes: inherent and apparent. The inherent optical properties (IOP) only depend on the constituents of the water, whereas the apparent optical properties (AOP) are not properties of the aquatic medium itself, although they do depend on its characteristics. Typical IOP are the absorption coefficient, the scattering coefficient or the scattering phase function. Some of the AOP include irradiance reflectance, attenuation coefficients or the average cosines [Pre76]. To obtain the in-water light field, 
we rely on the physically based theory of radiative transfer [Cha60], which relates the IOP and AOP. More precisely, the link is provided through the Radiative Transfer Equation (RTE) [SCP94], which takes into account emission, absorption and elastic scattering. Unfortunately this equation can not account for the phenomenon known as inelastic scattering described previously, which is of significant importance in ocean waters. We consequently expand the RTE by adding an extra term, thus obtaining the Full Radiative Transfer Equation (FRTE) [Gla95] and solving it by using an extended version of the method presented by Gutierrez et al. [GMAS05]:

$$
\begin{gathered}
\frac{\partial L\left(\lambda, \vec{\omega}_{o}\right)}{\partial x}=\alpha(\lambda) L_{e}\left(\lambda, \vec{\omega}_{o}\right)-\kappa(\lambda) L\left(\lambda, \vec{\omega}_{o}\right) \\
+\sigma(\lambda) \int_{\Omega} p\left(\lambda, \vec{\omega}_{i}, \vec{\omega}_{o}\right) L\left(\lambda, \vec{\omega}_{i}\right) d \vec{\omega}_{i} \\
+\int_{\Omega} \int_{W}\left\{\sigma\left(\lambda^{\prime}, \lambda\right) p\left(\lambda^{\prime}, \lambda, \vec{\omega}_{i}, \vec{\omega}_{o}\right) L\left(\lambda^{\prime}, \vec{\omega}_{i}\right)\right\} d \lambda^{\prime} d \vec{\omega}_{i}
\end{gathered}
$$

where $L$ is the radiance and $\vec{\omega}_{i}$ and $\vec{\omega}_{o}$ are, respectively, the incoming and outgoing directions of that radiance. $\alpha, \sigma$ and $\kappa$ are the absorption, scattering and extinction coefficients respectively. We assume $L_{e}\left(\lambda, \vec{\omega}_{o}\right)$ to be zero, thus making the medium non-emissive. Note that the last term models the inelastic scattering events and is expressed as a double integral over the domains of the solid angle $\Omega$ and wavelength $W$. Here $p\left(\lambda^{\prime}, \lambda, \vec{\omega}_{i}, \vec{\omega}_{o}\right)$ is the phase function for inelastic events and $\sigma\left(\lambda^{\prime}, \lambda\right)$ is the inelastic scattering function for the energy exchange between $\lambda^{\prime}$ and $\lambda$. For simplicity, when considering elastic interactions $\left(\lambda=\lambda^{\prime}\right)$ parameters $\lambda, \lambda^{\prime}$ are simplified to a single parameter $\lambda$. For processes such as fluorescence, where the photons are inelastic scattered to longer wavelengths, the function $\sigma\left(\lambda^{\prime}, \lambda\right)$ is usually expressed as:

$$
\sigma\left(\lambda^{\prime}, \lambda\right)=\alpha\left(\lambda^{\prime}\right) f\left(\lambda^{\prime}, \lambda\right)
$$

where $\alpha\left(\lambda^{\prime}\right)$ is the inelastic absorption coefficient and $f\left(\lambda^{\prime}, \lambda\right)$ is the wavelength redistribution function, which governs the efficiency of the energy transfer between wavelengths. It is defined as the probability of a photon of $\lambda^{\prime}$ that inelastically scatters being re-emitted at $\lambda$. Therefore, (2) expresses the inelastic scattering as a percentage of the inelastic absorption coefficient. Section 3.3 gives more details on how to model this redistribution function $f\left(\lambda^{\prime}, \lambda\right)$.

Our research on water simulation encompasses the fields of both computer graphics and oceanography, and it is free from the restrictions of previous works. The main contributions of this paper are:

- A compact, parameterized bio-optical model of ocean waters which can be used in computer graphics applications.

- A resolution method based on the theory of radiative transfer, which solves the FRTE by handling all kinds of inelastic scattering events and modeling both absorption and elastic scattering accurately. This method is based on photon mapping [Jen01].
- A link between the IOP of water and the resulting light field, which in turn defines its AOP, based on radiative transfer theory.

The remainder of this paper is organized as follows: Section 2 presents previous work on the simulation of light transport in water bodies. In Section 3 a comprehensive biooptical model is developed, whilst section 4 presents our simulation method. The paper ends with the results and conclusions.

\section{Related work}

The simulation of light transport in participating media usually either relies on Monte-Carlo techniques for ray tracing (Rushmeier and Torrance [RT87]; Nakamae et al. [NKON90]; Tadamura and Nakamae [TN95]) or attempts to solve the RTE, such as the method proposed by Kaneda et al. [KYNN91]. Nishita et al. [NSTN93] display water from outer space modifying this method, but both works only take into account single scattering. In the work of Premoze and Ashikhmin [PA01], no radiance due to scattering is calculated at all, using empirical equations based on experimental data instead. Mobley [Mob94] developed a method to solve the RTE analytically, but it cannot be extended to take into account inelastic scattering. Recently, the Lorenz-Mie theory has been generalized and applied to rendering natural waters by Frisvad, Christensen and Jensen [FCJ07], also neglecting the effects of inelastic scattering. Cerezo and Seron [CS04] also develop a bio-optical model. Whilst the goal of their work is closely related to ours, we overcome here significant shortcomings:

- They use a discrete ordinate method, which requires an angular and spatial discretization of the volume to be rendered. This imposes high memory requirements which seriously limit the complexity of the scenes that can be reproduced.

- In their work, inelastic scattering simulations are limited to fixed re-emissions in the $680 \mathrm{~nm}$. wavelength.

- They cannot provide a full solution to the light transport problem.

Gutierrez et al. [GMAS05] present a method that deals with participating media in which the index of refraction is not homogeneous, while also taking into account the simulation of some inelastic scattering events. They apply their method to the simulation of underwater imagery using a simplified, four-parameter model of ocean waters. In this regard, our paper offers improvement in the following ways:

- Our bio-optical model of ocean waters is more complete, thus making the simulations more accurate.

- They also fail to develop a complete description for the complex inelastic scattering events that occur underwater, and the method is limited to re-emissions at lower energy levels and at fixed wavelengths. In this paper all inelastic scattering events can be modeled, including Anti Stokes scattering events like Raman scattering (see Section 3.3). 
- We additionally offer simulations using real data from different seas as a means of visual validation.

\section{The Bio-Optical Model}

The various constituents of ocean water have a great influence in its optical properties. In order to solve the forward problem in ocean optics, the IOP have to be modeled and used in the FRTE. The values of these IOP can be obtained as the sum of the contributions of pure water and the dissolved particles and particulate matter present in the water, as proposed in [Mob94]. Optically pure water is devoid of any dissolved or suspended matter, and thus there is no scattering or absorption owed to particles or organic material [Mor74]. For saline pure water the salt concentration (35 to 39 parts per thousand) does influence the scattering and absorption functions. In particular it absorbs most wavelengths except for blue, with the absorption coefficient peaking at $760 \mathrm{~nm}$, and reaching a minimum at $430 \mathrm{~nm}$.

We develop our bio-optical model from three main IOP, with others like the extinction coefficient or the albedo derived from those three. These IOP are the absorption coefficient (3), the scattering coefficient (4) and the phase function (5), which for the elastic case can be written as (see Table 4 for a more detailed description of the functions used, including both the elastic and inelastic cases):

$$
\begin{aligned}
\alpha(\lambda) & =\alpha_{w}(\lambda)+\sum_{i} \alpha_{i}(\lambda) \\
\sigma(\lambda) & =\sigma_{w}(\lambda)+\sum_{i} \sigma_{i}(\lambda) \\
p(\lambda, \theta) & =\frac{\sigma_{w}(\lambda)}{\sigma(\lambda)} p_{w}(\lambda, \theta)+\sum_{i} \frac{\sigma_{i}(\lambda)}{\sigma(\lambda)} p_{i}(\lambda, \theta)
\end{aligned}
$$

where $\theta$ is the angle between the incoming $\vec{\omega}_{i}$ and outgoing $\vec{\omega}_{o}$ directions, the subscript $w$ stands for the contribution of the pure water (fresh or salty) and the subscript $i$ stands for the constituents in the water body such as biological particles or dissolved substances. We include three types of such constituents in our model, namely CDOM (Colored Dissolved Organic Matter, also know as yellow matter, present mainly in shallow ocean waters and harbors), phytoplankton (microscopic plants rich in chlorophyll) and minerals and organic detritus. The rest of this section will characterize the three main IOP (with elastic and inelastic scattering treated separately) for pure water and the three constituents. The next section will show how radiative transfer theory is applied to simulate the light field (which define the AOP) and render the final images.

\subsection{Modeling Absorption}

For the spectral absorption function of pure water $\alpha_{w}(\lambda)$ we rely on the work of Smith and Baker [SB81], whose tabulated values are well known in oceanography studies (shown in Table 1). Following further studies by Pope and
Fry [PF97], we use those values as an upper bound, to account for the fact that the true absorption can be, in fact, lower. The function shows that absorption is more prominent both in the UV and red ends of the spectrum. [PF97] also shows that absorption by salt in oceanic water is negligible. Based on the data by Bricaud, Morel and Prieur [BMP81], we model absorption by CDOM by fitting an exponential curve of the form:

$$
\alpha_{y}(\lambda)=\alpha_{y}\left(\lambda_{0}\right) e^{-S_{y}\left(\lambda-\lambda_{0}\right)}
$$

where the subscript $y$ denotes the constituent CDOM. $\lambda_{0}$ is a reference wavelength, often chosen to be $440 \mathrm{~nm}$ for yellow matter, and $S_{y}$ is the slope of the semilogarithmic absorption curve [Kir94]. $S_{y}$ is usually taken to be constant, with a value of $0.014 \mathrm{~nm}^{-1}$, but has been found to vary both geographically and temporally, and is also dependent on the wavelength range over which it is calculated [BMP81]. The values of absorption $\alpha_{y}\left(\lambda_{0}\right)$ at reference wavelengths also vary in a range between $0.01 \mathrm{~m}^{-1}$ to $20 \mathrm{~m}^{-1}$, as a function of turbidity [Kir94].

Phytoplankton absorbs a great amount of visible light, due to its chlorophyll pigment. The absorption function for chlorophyll peaks strongly at $430 \mathrm{~nm}$ and $670 \mathrm{~nm}$, being very weak in the mid range of the visible spectrum (thus the more phytoplankton the greener the hue of the water). The concentration of the chlorophyll in the water usually ranges from $0.01 \mathrm{mg} / \mathrm{m}^{3}$ for open waters to $100 \mathrm{mg} / \mathrm{m}^{3}$. The spectral absorption coefficient of the phytoplankton is usually expressed as a function of this concentration $C$ as:

$$
\alpha_{p}(\lambda)=C \alpha_{p}^{*}(\lambda)
$$

where $C$ can be defined as the concentration of the main pigment chlorophyll- $a\left(\mathrm{Chl}_{a}\right)$ or as the sum of the concentrations of $\mathrm{Chl}_{a}$ and its degradation products, the pheopigments. $\alpha_{p}^{*}$ is the specific spectral absorption coefficient (the absorption per unit of concentration) for a particular species of phytoplankton, given in $\mathrm{m}^{2} / \mathrm{mg}$. Typical values for specific absorptions of different species of phytoplankton can be found in the work of Sathyendranath, Lazzara and Prieur [SLP87] (see Table 1). A rough correspondence between chlorophyll concentrations and several oceanic water types is given by Morel [Mor88]. The absorption owed to organic detritus and minerals can be approximated by an exponential function, according to Roesler, Perry and Carder [RPC89]:

$$
\alpha_{d}(\lambda)=\alpha_{d}\left(\lambda_{0}\right) e^{-S_{d}\left(\lambda-\lambda_{0}\right)}
$$

Here the reference wavelength $400 \mathrm{~nm}$ is selected for $\lambda_{0}$ and typical values for the exponent coefficient $S_{d}$ will be in the range between $0.006 \mathrm{~nm}^{-1}$ to $0.014 \mathrm{~nm}^{-1}$, although 0.011 $\mathrm{nm}^{-1}$ is the most common value [RPC89]. Further studies confirm that the absorption spectra of minerals and detritus is well described by an exponential function with an average slope $S_{d}$ of $0.0123 \mathrm{~nm}^{-1}$, with slightly lower values than predicted at wavelengths below $440 \mathrm{~nm}$ [BSF*03]. 
Table 1: Absorption coefficient for a clear water body $\alpha_{w}$ (after Smith and Baker [SB81]) and specific absorption coefficient for phytoplankton $\alpha_{p}^{*}$ (after Sathyendranath, Lazzara and Prieur [SLP87]).

\begin{tabular}{cc|cccccccc}
\hline$\lambda$ & {$[\mathrm{nm}]$} & 380 & 440 & 500 & 550 & 610 & 670 & 720 & 780 \\
\hline$\alpha_{w}$ & {$\left[\mathrm{~cm}^{-1}\right]$} & 0.00022 & 0.000145 & 0.000257 & 0.000638 & 0.00289 & 0.0043 & 0.01169 & 0.0236 \\
$\alpha_{p}^{*}$ & {$\left[\mathrm{~m}^{2} \cdot \mathrm{mg}^{-1}\right]$} & 0.025 & 0.035 & 0.02 & 0.01 & 0.007 & 0.015 & 0.001 & 0.0001 \\
\hline
\end{tabular}

\subsection{Modeling Elastic Scattering}

For the pure water term we use the volume scattering function defined by the Einstein-Smoluchowski theory [Maz02], which models scattering at molecular level as small-scale fluctuations. Whilst usually Rayleigh's scattering is used instead, Einstein-Smoluchowski provides more accurate results, is well defined and imposes no overheads in the simulations. Its scattering coefficient and phase function are given by:

$$
\begin{aligned}
& \sigma_{w}(\lambda)=16.06 \beta_{w}\left(\lambda_{0}, 90^{\circ}\right)\left(\frac{\lambda_{0}}{\lambda}\right)^{4.32} \\
& p_{w}(\theta)=0.06225\left(1+0.835 \cos ^{2} \theta\right)
\end{aligned}
$$

Typical values for $\beta_{w}\left(\lambda_{0}, 90^{\circ}\right)$ for both fresh and saline pure water are given in [Mor74]. These values range from $14.1 \cdot 10^{-4} \mathrm{~m}^{-1}$ to $134.5 \cdot 10^{-4} \mathrm{~m}^{-1}$. All the scattering produced by CDOM has inelastic nature and thus will be described in next section.

Gordon and Morel [GM83] found that phytoplankton, even in small concentrations, also contribute to the total elastic scattering in the water. Its contribution is given by:

$$
\sigma_{p}(\lambda)=\left(\frac{550}{\lambda}\right) 0.30 C^{0.62}
$$

where the constant 0.30 is selected to fit the data collected from many types of waters. The actual upper bound for this constant has a value of 0.45 [GM83]. The phase function due to phytoplankton is given by an isotropic function ( $p_{p}=$ $1 / \pi)$.

The elastic scattering caused by organic detritus and minerals can be modeled based on Mie theory [GSO03]. The Henyey-Greenstein phase function models forward scattering fairly well but fails to reproduce backscattering with the same precision. We found that we can achieve a better fit by using a Two-Terms Henyey-Greenstein phase function (TTHG) [HG41]:

$$
p_{d}\left(\theta, \zeta, g_{f}, g_{b}\right)=\zeta p_{H G}\left(\theta, g_{f}\right)+(1-\zeta) p_{H G}\left(\theta, g_{b}\right)
$$

where $\zeta$ is a weighting function between zero and one. This common way of utilizing this combination defines a forward scattering lobe (first term), plus a backscattering lobe (second term), with $g_{f} \in[0 . .1]$ and $g_{b} \in[-1 . .0] . p_{H G}$ represents a simple Henyey-Greenstein phase function (HG):

$$
p_{H G}(\theta, g)=\frac{1-g^{2}}{\left(1+g^{2}-2 g \cos \theta\right)^{3 / 2}}
$$

The TTHG function not only models backscattering more precisely, but it can describe more complex particle scattering models, improving the fit at large and small angles as well. The shape of each of the two HG functions can be approximated by an ellipsoid, avoiding the relatively expensive exponent in its evaluation. The observation was first introduced by Schlick [BLSS93]. Due to the great variety of particulate matter, the scattering coefficient $\sigma_{d}$ can adopt a wide range of values. Table 2 shows typical values of this function (data after Stramski et al. [SBM01]).

\subsection{Modeling Inelastic Scattering}

For inelastic scattering, we need to model the possibility of an absorbed photon being re-emitted at a different wavelength. (2) includes a term $f\left(\lambda^{\prime}, \lambda\right)$ known as wavelength redistribution function, which represents the efficiency of the energy transfer between wavelengths. It is defined as the quotient between the energy of the emitted wavelength and the energy of the absorbed excitation wavelength, per wavelength unit. Reformulating in terms of photons instead of energy we have the spectral quantum efficiency function $\eta\left(\lambda^{\prime}, \lambda\right)$, defined as the ratio between the number of photons emitted at $\lambda$ per wavelength unit, and the number of absorbed photons at $\lambda^{\prime}$. Both functions are dimensional $\left(\mathrm{nm}^{-1}\right)$, and are related as follows:

$$
f\left(\lambda^{\prime}, \lambda\right)=\eta\left(\lambda^{\prime}, \lambda\right) \frac{\lambda^{\prime}}{\lambda}
$$

The wavelength redistribution function $f$, and therefore its associated spectral quantum efficiency function $\eta$, can be seen as a re-radiation matrix. A related dimensionless function that describes inelastic scattering is the quantum yield $\Gamma\left(\lambda^{\prime}\right)$, defined as the total number of photons emitted at all wavelengths divided by the number of photons absorbed at excitation wavelength $\lambda^{\prime}$. It is related to the spectral quantum efficiency function by:

$$
\Gamma\left(\lambda^{\prime}\right)=\int_{W} \eta\left(\lambda^{\prime}, \lambda\right) d \lambda
$$

The three functions $\Gamma\left(\lambda^{\prime}\right), f\left(\lambda^{\prime}, \lambda\right)$ and $\eta\left(\lambda^{\prime}, \lambda\right)$, depend on both the medium and the type of inelastic event. The two inelastic events with more influence in the in-water light field are fluorescence and Raman scattering. Phytoplankton and CDOM are important fluorescence sources, whilst Raman scattering is produced by pure water; minerals and detritus, on the other hand, do not produce any inelastic event. 
Table 2: Scattering coefficient for detritus $\sigma_{d t}$ and minerals $\sigma_{m}$ (After Stramski et al. [SBM01]).

\begin{tabular}{lll|cccccccc}
\hline & $\lambda$ & {$[\mathrm{nm}]$} & 380 & 440 & 500 & 550 & 610 & 670 & 720 & 780 \\
\hline detritus & $\sigma_{d t}$ & {$\left[\mathrm{~m}^{-1}\right]$} & 0.045 & 0.0375 & 0.0325 & 0.03 & 0.0285 & 0.0275 & 0.027 & 0.027 \\
minerals & $\sigma_{m}$ & {$\left[\mathrm{~m}^{-1}\right]$} & 0.0675 & 0.0525 & 0.05 & 0.045 & 0.04 & 0.036 & 0.034 & 0.032 \\
total & $\sigma_{d}$ & {$\left[\mathrm{~m}^{-1}\right]$} & 0.1125 & 0.09 & 0.0825 & 0.075 & 0.0685 & 0.0635 & 0.061 & 0.059 \\
\hline
\end{tabular}

\subsubsection{Fluorescence}

Fluorescence occurs when a molecule absorbs a photon of wavelength $\lambda^{\prime}$, and re-emits it at a longer wavelength $\lambda$ according to the fluorescence efficiency function $\eta_{F}\left(\lambda^{\prime}, \lambda\right)$. For the two main sources of fluorescence (phytoplankton and CDOM), re-emission follows an isotropic phase function. For phytoplankton, the wavelength of the re-emitted photons is independent of the excitation wavelength, although the intensity does show wavelength dependency [Mob94].

It is very common in ocean waters to see a color shift ranging from greenish to very bright green, or even yellowish. These hue shifts are mainly due to the variation in the concentration and type of the suspended microorganisms, specially phytoplankton and its related chlorophyll concentration, which presents an absorption function peaking at 350 $\mathrm{nm}$ and rapidly decaying to almost zero beyond $500 \mathrm{~nm}$. Only wavelengths between 370 and $690 \mathrm{~nm}$ can trigger fluorescence due to phytoplankton. This can be modeled as a dimensionless function $g_{p}\left(\lambda^{\prime}\right)$ so that:

$$
g_{p}\left(\lambda^{\prime}\right) \equiv \begin{cases}1 & \text { if } 370 \leq \lambda^{\prime} \leq 690 \mathrm{~nm} \\ 0 & \text { otherwise }\end{cases}
$$

The wavelength-independent quantum yield for phytoplankton $\Gamma_{p}\left(\lambda^{\prime}\right)$ ranges from 0.01 to 0.1 . Using (14) and (16), the relationship between the wavelength redistribution function $f_{p}\left(\lambda^{\prime}, \lambda\right)$ and the spectral quantum efficiency function $\eta_{p}\left(\lambda^{\prime}, \lambda\right)$ is:

$$
f_{p}\left(\lambda^{\prime}, \lambda\right)=\eta_{p}\left(\lambda^{\prime}, \lambda\right) \frac{\lambda^{\prime}}{\lambda} \equiv \Gamma_{p} g_{p}\left(\lambda^{\prime}\right) h_{p}(\lambda) \frac{\lambda^{\prime}}{\lambda}
$$

where $h_{p}(\lambda)$ is the fluorescence emission function per unit wavelength, and can be approximated by a gaussian [Mob94]:

$$
h_{p}(\lambda)=\frac{1}{\sqrt{2 \pi} \lambda_{\sigma}} \exp \left\{-\frac{\left(\lambda-\lambda_{0}\right)^{2}}{2\left(\lambda_{\sigma}\right)^{2}}\right\}
$$

$\lambda_{0}=685 \mathrm{~nm}$ is the wavelength of maximum emission and $\lambda_{\sigma}=10.6 \mathrm{~nm}$ represents the standard deviation. Using (7) and (17) we can now compute the inelastic scattering coefficient owed to phytoplankton $\sigma_{p}\left(\lambda^{\prime}, \lambda\right)$ following (2).

The other important source of fluorescence in water is CDOM. For relatively high concentrations of CDOM, its quantum yield $\Gamma_{y}\left(\lambda^{\prime}\right)$ varies between 0.005 and 0.025 . Following the work of Hawes [Haw92] we use the following formula to describe its spectral fluorescence quantum efficiency function:
Table 3: Water constituents and interactions

\begin{tabular}{lccc}
\hline Constituent & Absorption & Elastic Scat. & Inelastic Scat. \\
\hline $\begin{array}{l}\text { Pure water (w) } \\
\text { Minerals, detritus (d) }\end{array}$ & Yes & Yes & Raman Scattering \\
Phytoplankton (p) & Yes & Yes & No \\
CDOM (y) & Yes & No & $\begin{array}{c}\text { Fluorescence } \\
\text { Fluorescence }\end{array}$ \\
\hline & \\
$f_{y}\left(\lambda^{\prime}, \lambda\right)=A_{0}\left(\lambda^{\prime}\right) \exp \left\{-\left(\frac{\frac{1}{\lambda}-\frac{A_{1}}{\lambda^{\prime}}-B_{1}}{0.6\left(\frac{A_{2}}{\lambda^{\prime}}+B_{2}\right)}\right)^{2}\right\} \frac{\lambda^{\prime}}{\lambda}$
\end{tabular}

where $A_{0}, A_{1}, A_{2}, B_{1}$ and $B_{2}$ are empirical parameters whose values depend on the specific composition of the CDOM and can be found in [Mob94] (see Table 5). $A_{1}$ and $A_{2}$ are dimensionless, whereas the rest are given in $\mathrm{nm}^{-1}$. Like fluorescence due to phytoplankton, we can use (6) and (19) to compute the inelastic scattering coefficient $\sigma_{y}\left(\lambda^{\prime}, \lambda\right)$ following (2).

Our model can be easily extended to account for phosphorescence phenomena, which are intrinsically similar to fluorescence and are governed by the phosphorescence efficiency function. The only difference is that the re-emitted energy declines with time according to a function $d(t)$.

\subsubsection{Raman scattering}

Raman scattering influences the in-water light field, specially at great depths where sun irradiance becomes zero and only Raman radiance remains. It occurs when vibration and rotation in water molecules exchange energy with incoming photons, re-emitting them with approximately the same wavelength, but allowing for small shifts towards longer or shorter wavelengths. It can also be considered a spontaneous process. To isolate Raman inelastic events from fluorescence and other scattering events, it is usually studied in pure water, filtered several times, so that the second term in (4) becomes zero.

The Raman wavelength redistribution function $f_{w}\left(\lambda^{\prime}, \lambda\right)$ is usually described in terms of a sum of four Gaussian functions [Mob94]:

$$
f_{w}\left(\lambda^{\prime}, \lambda\right)=\frac{10^{7}}{\lambda^{\prime 2}} \frac{\sum_{j=1}^{4} A_{i} \frac{1}{\Delta \tilde{v}_{i}} \exp \left\{-\frac{\left[10^{7}\left(\frac{1}{\lambda^{\prime}}-\frac{1}{\lambda}\right)-\tilde{v}_{i}\right]^{2}}{\Delta \tilde{v}_{i}^{2}}\right\}}{\sqrt{\frac{\pi}{4 \ln 2}} \sum_{j=1}^{4} A_{j}}
$$

where $\tilde{\mathrm{v}}$ is the wavenumber $\left(\tilde{\mathrm{v}}=10^{7} / \lambda\right)$ given in $\mathrm{cm}^{-1}$. Typical parameter values $A_{i}, \tilde{\mathrm{v}}_{i}$ and $\Delta \tilde{\mathrm{v}}_{i}$ for the Raman redistribution function are given by Walrafen [Wal69] and are 
shown in Table 5. The inelastic scattering coefficient can now be obtained using $\alpha_{w}$ and $f_{w}$ in (2).

\section{The simulation method}

Having so far developed our bio-optical model, we can now formalize it into a set of parameters and equations to fully simulate the in-water light field. To summarize, the four constituents of the model and their interactions with light are given in Table 3. Table 4 shows how the main functions that define the model are derived from IOP and related functions at constituent level.

Table 4: The main functions of the model

\begin{tabular}{ll}
\hline Equations & \\
\hline$\alpha(\lambda)$ & $=\alpha_{d}(\lambda)+\alpha_{p}(\lambda)+\alpha_{w}(\lambda)+\alpha_{y}(\lambda)$ \\
$\sigma(\lambda)$ & $=\sigma_{w}(\lambda)+\sigma_{d}(\lambda)+\sigma_{p}(\lambda)$ \\
$p(\lambda, \theta)$ & $=\frac{\sigma_{w}(\lambda) p_{w}(\lambda, \theta)+\sigma_{d}(\lambda) p_{d}(\lambda, \theta)+\sigma_{p}(\lambda) p_{p}(\lambda, \theta)}{\sigma(\lambda)}$ \\
$\kappa(\lambda)$ & $=\alpha(\lambda)+\sigma(\lambda)$ \\
\hline$\alpha_{I}\left(\lambda^{\prime}\right)$ & $=\alpha_{p}\left(\lambda^{\prime}\right)+\alpha_{w}\left(\lambda^{\prime}\right)+\alpha_{y}\left(\lambda^{\prime}\right)$ \\
$p_{I}\left(\lambda^{\prime}, \lambda, \theta\right)$ & $=\frac{\alpha_{p}\left(\lambda^{\prime}\right) p_{p}\left(\lambda^{\prime}, \lambda, \theta\right)+\alpha_{w}\left(\lambda^{\prime}\right) p_{w}\left(\lambda^{\prime}, \lambda, \theta\right)+\alpha_{y}\left(\lambda^{\prime}\right) p_{y}\left(\lambda^{\prime}, \lambda, \theta\right)}{\alpha_{I}\left(\lambda^{\prime}\right)}$ \\
$f_{I}\left(\lambda^{\prime}, \lambda\right)$ & $=\frac{\alpha_{p}\left(\lambda^{\prime}\right) f_{p}\left(\lambda^{\prime}, \lambda\right)+\alpha_{w}\left(\lambda^{\prime}\right) f_{w}\left(\lambda^{\prime}, \lambda\right)+\alpha_{y}\left(\lambda^{\prime}\right) f_{y}\left(\lambda^{\prime}, \lambda\right)}{\alpha_{I}\left(\lambda^{\prime}\right)}$ \\
\hline
\end{tabular}

Table 5: Parameters of the model

\begin{tabular}{lccc}
\hline Parameter & Equations & Simulated values & Units \\
\hline$C$ & $(7)(11)$ & {$[0 . .1 .0]$} & $\frac{m g}{m^{3}}$ \\
$\alpha_{d}(400)$ & $(8)$ & {$[0 . .0 .1]$} & $m^{-1}$ \\
$\alpha_{y}(440)$ & $(6)$ & {$[0 . .0 .1]$} & $m^{-1}$ \\
\hline$S_{y}$ & $(6)$ & 0.014 & $\mathrm{~nm}^{-1}$ \\
$S_{d}$ & $(8)$ & 0.011 & $\mathrm{~nm}^{-1}$ \\
$A_{0}$ & $(19)$ & $\frac{150}{700}$ & $\mathrm{~nm}^{-1}$ \\
$A_{1}$ & $(19)$ & 4 & - \\
$A_{2}$ & $(19)$ & 4 & - \\
$B_{0}$ & $(19)$ & 1 & $\mathrm{~nm}^{-1}$ \\
$B_{1}$ & $(19)$ & $\overline{450 \cdot 10^{-7}}$ & $\mathrm{~nm}^{-1}$ \\
$\Gamma_{p}$ & $(17)$ & $650 \cdot 10^{-7}$ & - \\
$\Gamma_{y}$ & $(19)$ & 0.1 & - \\
$A_{i}, i=1 . .4$ & $(20)$ & $0.41,0.39,0.10,0.10$ & - \\
$\widetilde{\mathrm{v}}_{i}, i=1 . .4$ & $(20)$ & $3250,3425,3530,3625$ & - \\
$\Delta \widetilde{\mathrm{v}}_{i}, i=1 . .4$ & $(20)$ & $210,175,140,140$ & - \\
\hline
\end{tabular}

The model allows for easy adjusting of its parameters to simulate different types of water and thus obtain different in-water light fields. As well as minerals and detritus, other particulate components of water can be added from oceanographic studies (although minerals and detritus have the greatest influence in the final appearance of water). Mie theory can again be used to model the scattering by these new particles, and the phase function can be approximated by using a Two Terms Henyey-Greenstein phase function (12). An overview of the most significant parameters of the model, the equations in which they can be found and the corresponding values used for the simulations in this paper can be found in Table 5. Note that for simplicity we have not included the values that are already specified throughout the text during the explanation of the bio-optical model (more specifically, those included in tables 1 and 2). The first three correspond to the parameters analyzed in Figure 2.

Once we have formalized the model into a set of equations, we rely on radiative transfer theory to obtain a solution for the in-water light field. We solve the Full Radiative Transfer Equation (1) by extending the traditional photon mapping algorithm [Jen01] by taking into account all ten different events specified in Table 3, while allowing for both Stokes or anti-Stokes inelastic scattering. This enhancement is done in both stages: photon tracing and radiance estimation.

During the photon tracing stage in the original photon mapping method [Jen01], a Russian roulette algorithm is triggered at each interaction with the medium, deciding whether the photon is scattered or absorbed. In [GMAS05] the authors add a second Russian roulette which separates absorption from inelastic scattering; in the latter case, a new photon is generated at a different wavelength, but the algorithm considers just a single type of inelastic event with Stokes behavior. No anti-Stokes events are simulated. In contrast, our method uses just a single Russian roulette to choose between ten different kinds of interactions (including three types of inelastic events where the photons may gain or lose energy), and can be easily extended to handle an arbitrary number of different interactions. Finally, we improve the radiance estimation stage over previous methods by adding a term to take into account the contributions from the inelastic scattering events. The next subsections present the algorithm in more detail.

\subsection{Stage 1: Photon tracing}

We shoot photons from the light sources and let them interact with the geometry and the medium according to its optical distance, which is a function of the extinction coefficient (as in the original photon mapping method). We statistically decide at each interaction which type of event occurs (refer to Table 3) with just a single Russian roulette. At the interactions, photons are stored in a kd-tree as in traditional photon mapping.

The wavelength spectrum is box sampled into $N_{\lambda}$ samples, so absorption $(\alpha(\lambda))$ and scattering coefficients $(\sigma(\lambda))$ are implemented as $N_{\lambda}$-dimensional arrays while wavelength redistribution functions $\left(f\left(\lambda^{\prime}, \lambda\right)\right)$ are implemented as $N_{\lambda} \times N_{\lambda}$ square matrices. Each of the photons carries information about a portion of flux $(\Delta \Phi)$ at a certain sampled wavelength $\left(\lambda^{\prime}\right)$. Importance sampling is used for computing the optical distance, so $\Delta \Phi$ does not change along the photon tracing stage, while $\lambda^{\prime}$ changes for inelastic scattering events.

In order to apply the Russian roulette algorithm, we will define an albedo $\Lambda_{j}(\lambda)$ for each interaction $j$ as follows:

- If interaction $j$ represents an elastic scattering event, then $\Lambda_{j}(\lambda)=\frac{\sigma_{j}(\lambda)}{\kappa(\lambda)}$

- If $j$ represents an absorption interaction that does not 
show inelastic scattering (detritus and minerals, basically), then $\Lambda_{j}(\lambda)=\frac{\alpha_{j}(\lambda)}{\kappa(\lambda)}$

- For each absorption interaction that could generate inelastic scattering (pure water, phytoplankton and CDOM) we define its inelastic probability $\left(\chi_{j}\right)$, the probability that an absorption event generates an inelastic scattering event:

$$
\chi_{j}\left(\lambda^{\prime}\right)=\int_{\lambda_{a}}^{\lambda_{b}} f_{I}\left(\lambda^{\prime}, \lambda\right) d \lambda \approx \sum_{i=1}^{N_{\lambda}} f_{I}\left(\lambda^{\prime}, \lambda_{i}\right)
$$

where $\lambda_{a}$ and $\lambda_{b}$ are the lower and upper limits of the simulated wavelengths, and $i \in\left[1 . . N_{\lambda}\right]$ refer to samples in wavelength domain:

- If interaction $j$ represents the effective inelastic scattering event within the absorption interaction: $\Lambda_{j}(\lambda)=$ $\frac{\alpha_{j}(\lambda)}{\kappa(\lambda)} \chi_{j}(\lambda)$

- If interaction $j$ represents the pure absorption event (no inelastic scattering happening at all): $\Lambda_{j}(\lambda)=$ $\frac{\alpha_{j}(\lambda)}{\kappa(\lambda)}\left(1-\chi_{j}(\lambda)\right)$

Thus, at each interaction a random number $\xi$ between 0 and 1 is generated resulting in (between parenthesis, example values of $\Lambda_{j}$ at $\lambda=500 \mathrm{~nm}$ that determine the size of the corresponding interval are included):

- $\xi_{\varepsilon}\left[0, \xi_{1}\right) \rightarrow$ absorption by pure water $\left(2.51 \cdot 10^{-1}\right)$.

- $\xi \varepsilon\left[\xi_{1}, \xi_{2}\right) \rightarrow$ Raman scattering, inelastic scattering by pure water $\left(1.21 \cdot 10^{-9}\right)$.

- $\xi \varepsilon\left[\xi_{2}, \xi_{3}\right) \rightarrow$ absorption by minerals and detritus (7.12 . $\left.10^{-2}\right)$.

- $\xi \varepsilon\left[\xi_{3}, \xi_{4}\right) \rightarrow$ absorption by phytoplankton $\left(4.90 \cdot 10^{-3}\right)$.

- $\xi \varepsilon\left[\xi_{4}, \xi_{5}\right) \rightarrow$ inelastic scattering by phytoplankton (2.18 . $\left.10^{-3}\right)$.

- $\xi \varepsilon\left[\xi_{5}, \xi_{6}\right) \rightarrow$ absorption by CDOM $\left(7.83 \cdot 10^{-2}\right)$.

- $\xi \varepsilon\left[\xi_{6}, \xi_{7}\right) \rightarrow$ inelastic scattering by CDOM $\left(1.21 \cdot 10^{-2}\right)$.

- $\xi_{\varepsilon}\left[\xi_{7}, \xi_{8}\right) \rightarrow$ elastic scattering by pure water $\left(7.44 \cdot 10^{-3}\right)$

- $\xi \varepsilon\left[\xi_{8}, \xi_{9}\right) \rightarrow$ elastic scattering by minerals and detritus $\left(2.94 \cdot 10^{-1}\right)$.

- $\xi \varepsilon\left[\xi_{9}, 1\right] \rightarrow$ elastic scattering by phytoplankton (2.79 . $\left.10^{-1}\right)$.

where $\xi_{i}(\lambda)$ is given by $\xi_{i}(\lambda)=\sum_{j=1}^{i} \Lambda_{j}(\lambda)$

To compute the new re-emitted wavelength after a inelastic scattering event $i$, the normalized wavelength redistribution function $\frac{f_{i}\left(\lambda^{\prime}, \lambda\right)}{\chi_{i}\left(\lambda^{\prime}\right)}$ is treated as a probability distribution function (PDF) given the excitation wavelength $\lambda^{\prime}$. To sample it efficiently we first build its normalized cumulative distribution function (CDF) and then inverse importance sample this CDF. Greater values of the PDF for a given wavelength will translate to steeper areas of the CDF, thus increasing the probability of a re-emission at such wavelength. Note that the definition of $f_{i}\left(\lambda^{\prime}, \lambda\right)$ is not limited to the visible spectrum, which might result in re-emissions happening at wavelengths beyond the visible spectrum. However, as $\chi_{i}\left(\lambda^{\prime}\right)$ is limited to the simulated (visible) spectrum, only inelastic interactions within this spectrum are considered. It could happen that a photon inelastically scattered at such wavelengths suffers a second inelastic scattering event that brings it back to the visible light range. Given the low probability of this chain of events and our computer graphics approach, we assume that a photon beyond the visible spectrum is definitely absorbed. Figure 1 shows a global overview of the algorithm during the photon tracing stage.

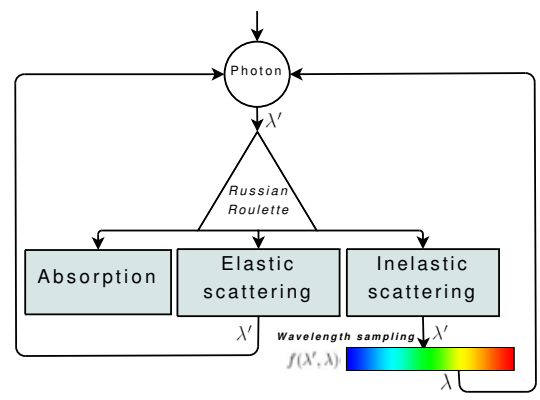

Figure 1: Photon tracing algorithm. Inelastic scattering events generate a photon with a different associated wavelength according to the wavelength redistribution function.

\subsection{Stage 2: radiance estimate}

To estimate radiance we adopt a tradeoff between speed and memory requirements similar to the proposed by Jensen and Christensen [JC98]: we only store photons in the photon map if they have been reflected or transmitted from surfaces, or if they have already been scattered at least once. Thus, we can compute single scattering more efficiently by ray marching through the medium and sampling the light sources by casting shadow rays. Taking into account the wavelength redistribution function for inelastic scattering, a new addend will be added at each step of the ray marching process:

$$
\sum_{l=1}^{N} \sum_{i=1}^{N_{\lambda}}\left\{L_{l}\left(\lambda_{i}^{\prime}, \vec{w}_{l}\right) p_{I}\left(\lambda_{i}^{\prime}, \lambda, \vec{w}_{l}, \vec{w}_{o}\right) \alpha_{I}\left(\lambda_{i}^{\prime}\right) f_{I}\left(\lambda_{i}^{\prime}, \lambda\right) \Delta x\right\}
$$

where $i \in\left[1 . . N_{\lambda}\right]$ and $l \in[1 . . N]$ refer to samples in the wavelength and light source domain respectively, $\vec{w}_{l}$ is the direction to the light with an incoming radiance $L_{l}$ and $\Delta x$ represent the ray marching steps.

Multiple scattering will be computed from the photon map, finding in the kd-tree the $n$ photons which are closest to the estimation point by using the typical nearest neighbours algorithm. To account for multiple inelastic scattering we modify the radiance estimate expression of [JC98] by including a new term:

$$
\sum_{k=1}^{n}\left\{p_{I}\left(\lambda_{k}^{\prime}, \lambda, \vec{w}_{k}, \vec{w}_{o}\right) f_{I}\left(\lambda_{k}^{\prime}, \lambda\right) \frac{\Delta \Phi_{k}}{\frac{4}{3} \pi r^{2}}\right\}
$$

where $r$ is the radius of the sphere that contains the $n$ closest photons, and $k$ represents each of the stored photons.

\section{Results}

We have used the values from Table 5 for our simulations. In the images produced we only vary the chlorophyll concentration $C$, minerals and detritus turbidity $\alpha_{d}(400)$ and 


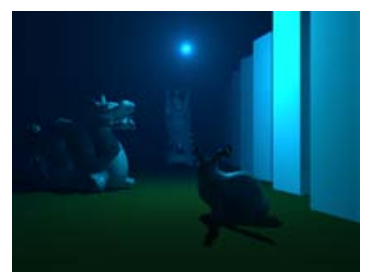

(a) $C=0, \alpha_{d}=0, \alpha_{y}=0$

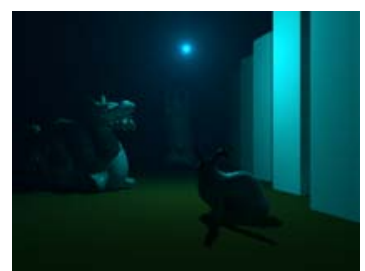

(e) $C=0, \alpha_{d}=0.1, \alpha_{y}=0$

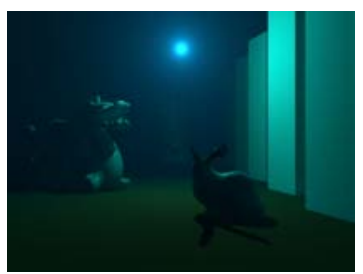

(i) $C=0, \alpha_{d}=0, \alpha_{y}=0.1$

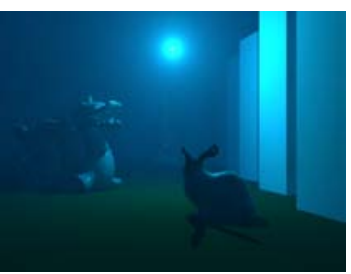

(b) $C=0.01, \alpha_{d}=0, \alpha_{y}=0$

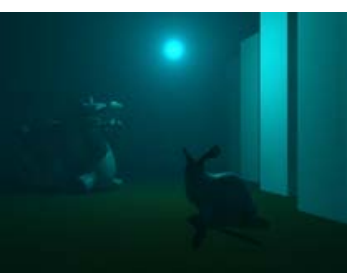

(f) $C=0.01, \alpha_{d}=0.1, \alpha_{y}=0$

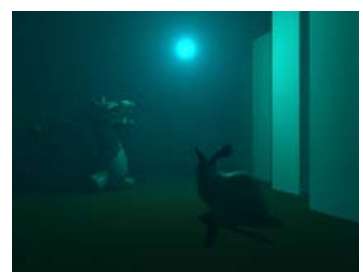

(j) $C=0.01, \alpha_{d}=0, \alpha_{y}=0.1$

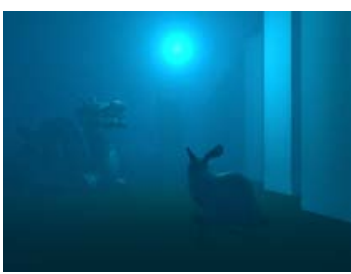

(c) $C=0.1, \alpha_{d}=0, \alpha_{y}=0$

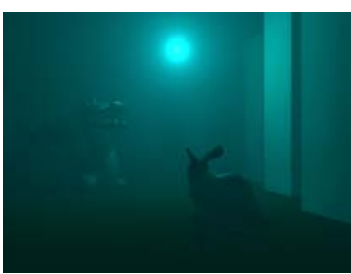

(g) $C=0.1, \alpha_{d}=0.1, \alpha_{y}=0$

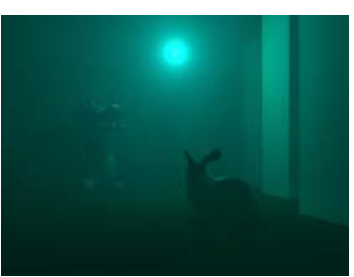

(k) $C=0.1, \alpha_{d}=0, \alpha_{y}=0.1$

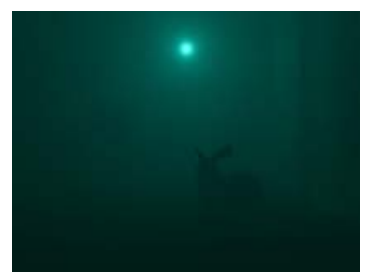

(d) $C=1, \alpha_{d}=0, \alpha_{y}=0$

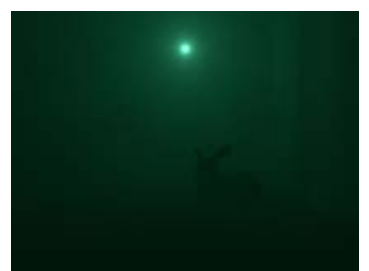

(h) $C=1, \alpha_{d}=0.1, \alpha_{y}=0$

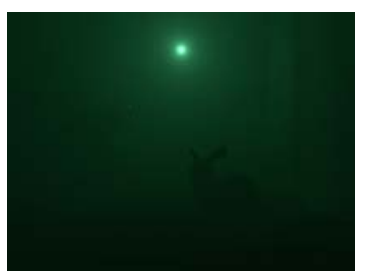

(1) $C=1, \alpha_{d}=0, \alpha_{y}=0.1$

Figure 2: Resulting pictures varying the chlorophyll concentration $C$, the minerals and detritus turbidity $\alpha_{d}$ at $400 \mathrm{~nm}$ and the CDOM turbidity $\alpha_{y}$ at $440 \mathrm{~nm}$.

CDOM turbidity $\alpha_{y}(440)$. The choice of those three parameters to reduce the dimensionality of the model was based on their greater overall influence on the resulting light field. The photon map contains 400000 photons, with 250 used in the estimation of radiance. Ray-marching depth is set at 200 steps. Each of the images has been rendered in a Dual Xeon Pentium 4 at $2.8 \mathrm{GHz}$ with $2 \mathrm{~GB}$ RAM at $512 \times 384$ resolution, casting one ray per pixel, and took approximately 20 minutes to render. This time is roughly independent of the number of parameters of the bio-optical model. In order to reduce these computation times, several optimization techniques could be adopted, like using adaptive ray-marching or radiance caching strategies [JDZJ08]. Additionally, perceptual issues could be taken into account, using just an approximate solution in areas of the image where the error is known to be perceptually negligible [SGA*07].

Energy balances show that on average almost $99 \%$ of the energy emitted by the light sources is absorbed after just a few interactions of the photons, with very incremental variation after the fourth interaction and negligible contribution after the fifth. This relatively fast convergence is due to the strong absorption in water. We have therefore limited the number of interactions per photon to five, in order to speed up the simulations. Variations of the parameters $C, \alpha_{d}(400)$ and $\alpha_{y}(440)$ yield different probabilities for absorption, elastic and inelastic scattering events, which in turn affect the in-water light field. The results can be seen in Figure 2, with each of the varying parameters influencing the final light field as follows:

- Chlorophyll concentration $(C)$ affects mainly both elastic and inelastic scattering. The effects of inelastic scattering are mostly masked by the more predominant elastic scattering and absorption, which increases slowly. The third column in Figure 2 shows brighter images than the previous two due to in-scattering. For higher values (fourth column), out-scattering prevails and the images become darker.

- Minerals and detritus turbidity $\left(\alpha_{d}(400)\right)$ increases absorption at lower wavelengths, thus reducing the brightness of the scene and the overall blue hue. Scattering is also increased, making the images appear murkier. Figure 2 shows variations of the minerals and detritus turbidity between the first and second rows for direct comparison.

- CDOM turbidity $\left(\alpha_{y}(440)\right)$ slightly increases absorption (darker images) and introduces inelastic scattering (change in hue). This can be seen by comparing the first and third rows in Figure 2.

We have undergone a visual validation of our model by rendering different natural waters. Figure 3 shows the resulting underwater images for Atlantic, Mediterranean, Baltic, North Sea and shallow coastal waters rich in CDOM respectively. All the images have been simulated at the same depth 

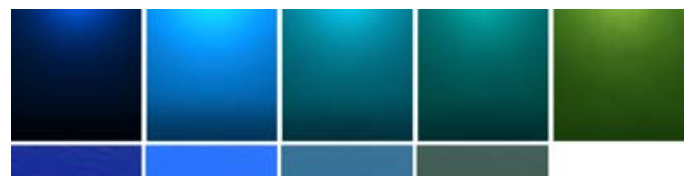

Figure 3: Rendered images of different waters. From left to right: Atlantic, Mediterranean, Baltic, North Sea and shallow coastal waters rich in CDOM. Smaller patches below for comparison purposes by Frisvad et al. [FCJ07] (used with permission).

and are illuminated by the same isotropic point light source. The changes in color are clearly noticeable, from a darker blue in the case of Atlantic water, to the greener hue in the image of the North Sea. The smaller patches below the first four images correspond to the simulations by Frisvad et al. [FCJ07] for the same types of water, and are shown for comparison purposes. Our simulations based on radiative transfer approximately match their simulations based on LorenzMie theory. The differences are mainly owed to two factors: on the one hand, the overall darker tone in our images is due to in-water absorption, whereas [FCJ07] renders the surface of the water body; on the other hand, the absence of inelastic scattering effects in [FCJ07] can have a visible influence the final appearance of water, as shown in Figure 4 for the Baltic case. The properties of the water have been adjusted according to measurements found in [BSF*03] [Mob94] for our bio-optical model and [BSF*03] in the model by Frisvad et al. In both cases, it is only the changes in the constituents of the waters which yield the different colors. We have additionally performed a numerical analysis of the in-water radiance field, to quantify the influence of each constituent. The results can be seen in Figure 5.

\section{Conclusion}

We have presented a complete bio-optical model of ocean water based on parameterizing its intrinsic optical properties. Relying on radiative transfer theory, we obtain the resulting in-water light field by extending the rendering algorithm presented in [GMAS05]. The extension can now handle more complex interactions between light and water, including inelastic scattering with anti-Stokes behavior, where the scattered photon absorbs energy from the medium and is re-emitted at higher energies. We have additionally studied the influence of the parameters in the apparent optical properties of water in the scene, which are defined by the light field obtained. We have performed an energy-balance analysis, and visual validation of the method has been provided by direct comparison with images by Frisvad et al. [FCJ07], rendering different types of waters based on published constituent data.

We have included Raman scattering by pure water and fluorescence by phytoplankton and CDOM as inelastic scattering events with energy transfers. Even though their combined quantitative contribution to the overall radiance field is usually less than $2 \%$ (see Figure 5), this relatively small percentage does have a clear influence on the apparent optical properties, as Figure 4 shows. We thus argue that these events, usually overlooked in computer graphics literature, are qualitatively important for underwater imagery and should be included in a complete simulation. Other types of inelastic scattering such as Compton, Bragg or Brillouin could also be added, although their influence is more incremental. Other particulate elements could be easily added as well just by including their corresponding absorption and scattering coefficients in the model; however, the three constituents treated here (phytoplankton, minerals and detritus and $\mathrm{CDOM}$ ) have the most influence in the final radiance field.

The results show how the model developed can easily be used for physically-based simulations of underwater imagery. We believe this work can be of interest not only in the computer graphics community, but in remote sense or oceanographic studies as well.
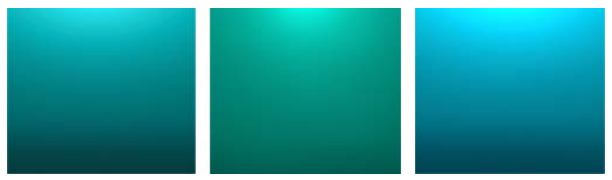

Figure 4: The influence of inelastic scattering in the apparent optical properties of water (Baltic sea): Left, no inelastic scattering. Center, just chlorophyll inelastic scattering (as in [GMASO5]). Right, all inelastic scattering events included in the simulation.

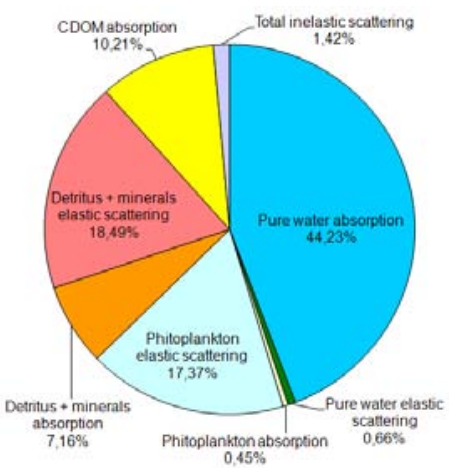

Figure 5: Radiance distribution of the resulting in-water light field per type of event (Baltic Sea).

\section{Acknowledgments}

We thank the anonymous reviewers for their valuable comments. This research was partly done under the sponsorship of the Spanish Ministry of Education and Research through the projects TIN2004-07672-C03-03 and TIN2007-63025.

\section{References}

[BLSS93] Blasi P., Le SAEC B., Schlick C.: A rendering algorithm for discrete volume density objects. Computer Graphics Forum (Eurographics 93) 12, 3 (1993), 201-210. 4 
[BMP81] Bricaud A., Morel A., Prieur L.: Absorption by dissolved organic matter of the sea (yellow substance) in the uv and visible domains. Limnol. Oceanogr. 26, 1 (1981), 43-53. 3

[BSF*03] Babin M., Stramski D., Ferrari G. M., ClausTre H., Bricaud A., Obolensky G., HoepfFner N.: Variations in the light absorption coefficients of phytoplankton, nonalgal particles, and dissolved organic matter in coastal waters around europe. J. Geophys. Res. 108(C7), 3211 (2003). 3, 9

[Cha60] Chandrasekhar S.: Radiative Transfer. Dover Publications, Inc., 1960. 2

[CS04] CEREzo E., SERON F. J.: Rendering natural waters taking fluorescence into account: Research articles. Comput. Animat. Virtual Worlds 15, 5 (2004), 471-484. 2

[FCJ07] Frisvad J. R., Christensen N. J., Jensen H. W.: Computing the scattering properties of participating media using Lorenz-Mie theory. ACM Trans. Graph. 26, 3 (2007), 60. 2, 9

[Gla95] Glassner A. S.: Principles of Digital Image Synthesis. Morgan Kaufmann Publishers Inc., San Francisco, CA, USA, 1995. 2

[GM83] Gordon H. R., Morel A.: Remote Assessment of Ocean Color for Interpretation of Satellite Visible Imagery: A Review, vol. 4 of Lecture Notes on Coastal and Estuarine Studies. Springer-Verlag, New York, 1983. 4

[GMAS05] Gutierrez D., Munoz A., Anson O., Serón F. J.: Non-linear volume photon mapping. In Proc. of the Eurographics Symposium on Rendering Techniques, Konstanz, Germany, June 29 - July 1, 2005 (2005), pp. 291-300. 2, 6, 9

[GSO03] Green R. E., SosiK H. M., Olson R. J.: Contributions of phytoplankton and other particles to inherent optical properties in new england continental shelf waters. Limnol. Oceanogr. 48, 6 (2003), 2377-2391. 4

[Haw92] HAWES S.: Quantum fluorescence efficiencies of marine fulvic and humid acids. PhD thesis, Dept. of Marince Science, Univ. of South Florida, 1992. 5

[HG41] Henyey L., Greenstein J.: Diffuse radiation in the galaxy. Astrophysics Journal 93 (1941), 70-83. 4

[JC98] Jensen H. W., Christensen P. H.: Efficient simulation of light transport in scenes with participating media using photon maps. In SIGGRAPH 98 Conference Proceedings (jul 1998), Cohen M., (Ed.), Annual Conference Series, ACM SIGGRAPH, Addison Wesley, pp. 311-320. 7

[JDZJ08] Jarosz W., Donner C., ZWICKer M., JEnSEN H. W.: Radiance caching for participating media. To appear in ACM Transactions on Graphics (2008). 8

[Jen01] JENSEN H. W.: Realistic image synthesis using photon mapping. A.K. Peters, Natick, Massachussets, 2001. 2, 6

[Kir94] KIRK J. T.: Light and photosynthesis in aquatic ecosystems. Cambridge University Press, New York, 1994. 3

[KYNN91] KAneda K., YuAn G., NAKAmaE E., Nishita T.: Realistic visual simulation of water surfaces taking into account radiative transfer. In Proc. of CAD/Graphics 91 (1991), pp. 2530. 2

[Maz02] Mazo R. M.: Brownian Motion: Fluctuations, Dynamics and Applications, vol. 112 of International Series of Monographs on Physics. Oxford University Press Inc., Great Claren- don Street, Oxford, 2002, ch. Einstein-Smoluchowski Theory, pp. 46-62. 4

[Mob94] Mobley C. D.: Light and Water: Radiative Transfer in Natural Waters. Academic Press, Inc., San Diego, 1994. 1, 2, 3 , 5,9

[Mor74] Morel A.: Optical Aspects of Oceanography. Academic Press, New York, 1974. 3, 4

[Mor88] MOREL A.: Optical modeling of the upper ocean in relation to its biogenous matter content (case i waters). Journal of Geophysical Research 93, C9 (1988), 10749-10768. 3

[NKON90] NaKamae E., Kaneda K., OKamoto T., NishitA T.: A lighting model aiming at drive simulators. Computer Graphics 24, 4 (Aug. 1990), 395-404. 2

[NSTN93] Nishita T., Shirai T., Tadamura K., Nakamae E.: Display of the earth taking into account atmosphere scattering. In Computer Graphics (SIGGRAPH '93 Proceedings) (1993), vol. 24, pp. 175-182. 2

[PA01] PREMOZE S., Ashikhmin M.: Rendering natural waters. Comput. Graph. Forum 20, 4 (2001), 189-199. 2

[PF97] POPE R. M., Fry E. S.: Absorption spectrum (380-700 $\mathrm{nm}$ ) of pure water. ii. integrating cavity measurements. Applied Optics 36, 33 (1997), 8710-8723. 3

[Pre76] Preisendorfer R. W.: Introduction, vol. 1 of Hydrologic Optics. National Technical Information Service, Springfield, IL, 1976. 1

[RPC89] Roesler C. S., Perry M. J., CARder K. L.: Modeling in situ phytoplankton absorption from total absorption spectra in productive inland marine waters. Limnol. Oceanogr. 34, 8 (1989), 1510-1523. 3

[RT87] RushmeIER H. E., TORRANCE K. E.: The zonal method for calculating light intensities in the presence of a participating medium. Computer Graphics 21, 4 (July 1987), 293-302. 2

[SB81] SMith R. C., BAKER K. S.: Optical properties of the clearest natural waters (200-800 nm). Appl. Opt. 20 (1981), 177184. 3,4

[SBM01] Stramski D., Bricaud A., Morel A.: Modeling the inherent optical properties of the ocean based on the detailed composition of planktonic community. Applied Optics 40 (2001), 2929-2945. 4, 5

[SCP94] Spinrad R. W., CARder K. L., Perry M. J. (Eds.): Ocean Optics. No. 25 in Oxford Monographs on Geology and Geophysics. Oxford University Press, 1994. 2

[SGA*07] Sundstedt V., Gutierrez D., Anson O., BAnTERle F., Chalmers A.: Perceptual rendering of participating media. ACM Transactions of Applied Perception 4, 3 (2007). 8

[SLP87] Sathyendranath S., LazZara L., Prieur L.: Variations in the spectral values of specific absorption of phytoplankton. Limnol. Oceanogr. 32, 2 (1987), 403-415. 3, 4

[TN95] TADAMURA K., NAKAMAE E.: Computer Graphics: Developments in Virtual Environments. Academic Press, 1995, ch. Modeling the colour of Water in Lightning Design, pp. 97114. 2

[Wal69] WalRafen G. E.: Continuum model of water-an erroneous interpretation. Journal of Chemical Physics 50, 1 (January 1969), 567-569. 5 